\title{
Investigations on birefringence effects in polymer optical fiber Bragg gratings
}

\author{
X. Hu*a , D. Saez-Rodriguez ${ }^{\mathrm{b}}$, O. Bang ${ }^{\mathrm{c}}$, D. J. Webb ${ }^{\mathrm{b}}$, C. Caucheteur ${ }^{\mathrm{a}}$ \\ ${ }^{a}$ Electromagnetism and Telecommunication Department, University of Mons (UMONS), \\ Boulevard Dolez, 31, 7000 Mons, Belgium; \\ ${ }^{\mathrm{b}}$ Aston Institute of Photonic Technologies, Aston University, Birmingham, B4 7ET, UK; \\ ${ }^{c}$ Deprtment of Photonics Engineering, Technical University of Denmark, Ørsteds Plads, 2800, Kgs. \\ Lyngby, Denmark
}

\begin{abstract}
Step-index polymer optical fiber Bragg gratings (POFBGs) and microstructured polymer optical fiber Bragg gratings (mPOFBGs) present several attractive features, especially for sensing purposes. In comparison to FBGs written in silica fibers, they are more sensitive to temperature and pressure because of the larger thermo-optic coefficient and smaller Young's modulus of polymer materials. (M)POFBGs are most often photowritten in poly(methylmethacrylate) (PMMA) materials using a continuous-wave $325 \mathrm{~nm} \mathrm{HeCd}$ laser. For the first time to the best of our knowledge, we study photoinduced birefringence effects in (m)POFBGs. To achieve this, highly reflective gratings were inscribed with the phase mask technique. They were then monitored in transmission with polarized light. For this, (m)POF sections a few $\mathrm{cm}$ in length containing the gratings were glued to angled silica fibers. Polarization dependent loss (PDL) and differential group delay (DGD) were computed from the Jones matrix eigenanalysis using an optical vector analyser. Maximum values exceeding several $\mathrm{dB}$ and a few picoseconds were obtained for the PDL and DGD, respectively. The response to lateral force was finally investigated. As it induces birefringence in addition to the photo-induced one, an increase of the PDL and DGD values were noticed.
\end{abstract}

Keywords: Polymer optical fiber, Bragg grating, birefringence, transverse force, sensor

\section{INTRODUCTION}

Fiber Bragg gratings (FBGs) were first fabricated in polymer optical fibers (POFs) in $1999^{1}$. Since then, Polymer optical FBGs have been photo-inscribed point-by-point ${ }^{2}$ and with the phase mask technique, including single exposure ${ }^{3-5}$ and laser beam scanning ${ }^{6,7}$. FBGs inscribed in POFs present several attractive features for sensing purposes. Compared to their counterparts produced in silica fibers, they are more sensitive to temperature and pressure because of the larger thermo-optic coefficient and smaller Young's modulus of polymer materials ${ }^{8-13}$. Besides, PMMA demonstrates absorption of moisture up to 2 w.t. \%, so that PMMA FBGs can be used as humidity sensors ${ }^{14}$, biochemical concentration sensors ${ }^{15}$ or water detection sensors ${ }^{16}$. Although different polymer materials can be used to manufacture $\mathrm{POFs}^{17-19}$, poly(methyl methacrylate) (PMMA) is the most often encountered one.

The step-index POFs used in this work were supplied by the Hong Kong Polytechnic University. The cladding is in pure PMMA while the core is composed of PMMA doped with diphenyl sulfide (5\% mole) and trans-4-stilbenemethanol ( $1 \%$ w.t.). The mPOFs were supplied by the Technical University of Denmark, and were produced using undoped PMMA with a hexagonal structure of three rings in the inner cladding. $6 \mathrm{~mm}$ long step-index POFBGs and $5 \mathrm{~mm}$ long mPOFBGs with high reflectivity were photo-inscribed thanks to a helium-cadmium laser emitting at $325 \mathrm{~nm}$ with the scanning phase mask technique $\mathrm{e}^{20-22}$.

In this paper, for the first time to the best of our knowledge, we studied photo-induced birefringence effects in (m)POFBGs arising from both the lateral inscription process and the application of a transverse force. After photoinscription, (m)POF sections with gratings were UV-glued or connected to silica fibers on both sides of (m)POFs, and then birefringence effects were monitored in transmission with polarized light.

*xuehao.hu@umons.ac.be

Micro-structured and Specialty Optical Fibres III, edited by Kyriacos Kalli,

Alexis Mendez, Proc. of SPIE Vol. 9128, 91280Q · C 2014 SPIE

CCC code: $0277-786 X / 14 / \$ 18 \cdot$ doi: $10.1117 / 12.2054025$

Proc. of SPIE Vol. $912891280 Q-1$ 
Like in silica fiber, the lateral writing process in (m)POF induces a small quantity of birefringence that combines with the intrinsic fiber birefringence and leads to polarization effects within the grating ${ }^{23}$. This small amount of birefringence causes the orthogonal polarization modes to experience different couplings through the grating ${ }^{24}$. In the presence of birefringence, the resulting transmitted spectrum is then the combination of two overlapping, offset but otherwise identical spectra. Because these two spectra cannot be generally distinguished due to the limited resolution of the measurement devices, two measurements of polarization dependent loss (PDL) and differential group delay (DGD) $)^{25,26}$ were made. They were both computed from the Jones matrix eigenanalysis method using an optical vector analyzer (OVA CTe from Luna Technologies, attesting that the photo-induced birefringence resulting from the side inscription process is $7 \times 10^{-6}$ for the step-index $\mathrm{POFs}^{27}$.

Finally, the response to lateral force in the range $(0-0.75 \mathrm{~N})$ was investigated on gratings written in step-index POFs with an experimental set-up allowing the application of controlled transverse loads on the gratings. As it induces mechanical birefringence in addition to the photo-induced one, a drastic increase of the PDL was noticed.

\section{EXPERIMENTAL SET-UP}

The step-index POFBGs were manufactured at the University of Mons. The laser used in this work is a He-Cd laser (Kimmon IK5751I-G) with an output power of $30 \mathrm{~mW}$ at $325 \mathrm{~nm}$. The output beam width of the laser is $1.2 \mathrm{~mm}$. Figure 1 depicts the experimental set-up. The inox tube positioned above the laser prevented air turbulence caused by the cooling system perturbing the photo-writing process. The UV beam emitted by the laser was reflected by four mirrors (even number of reflections to preserve the output state of polarization) towards the POF, which was held in a V-groove plate. A uniform phase mask (Coherent) with a period of $1044 \mathrm{~nm}$ was placed above the fiber as closely as possible. A cylindrical lens with $10 \mathrm{~cm}$ focal length was used upstream of the phase mask to focus the UV beam on the fiber core. The movement of the third mirror was controlled by a motor-driven translation stage, which was used to scan the laser along the fiber over a maximum length of $20 \mathrm{~mm}$. Amplitude reflected spectrum measurements were obtained with an FBG interrogator (FS2200SA from FiberSensing), which presents a wavelength resolution of $1 \mathrm{pm}$ and a scanning rate of $1 \mathrm{~Hz}$. MPOFBGs were fabricated at Aston University using a He-Cd laser (Kimmon IK3301R-G) and the experimental set-up is similar to the one described above.

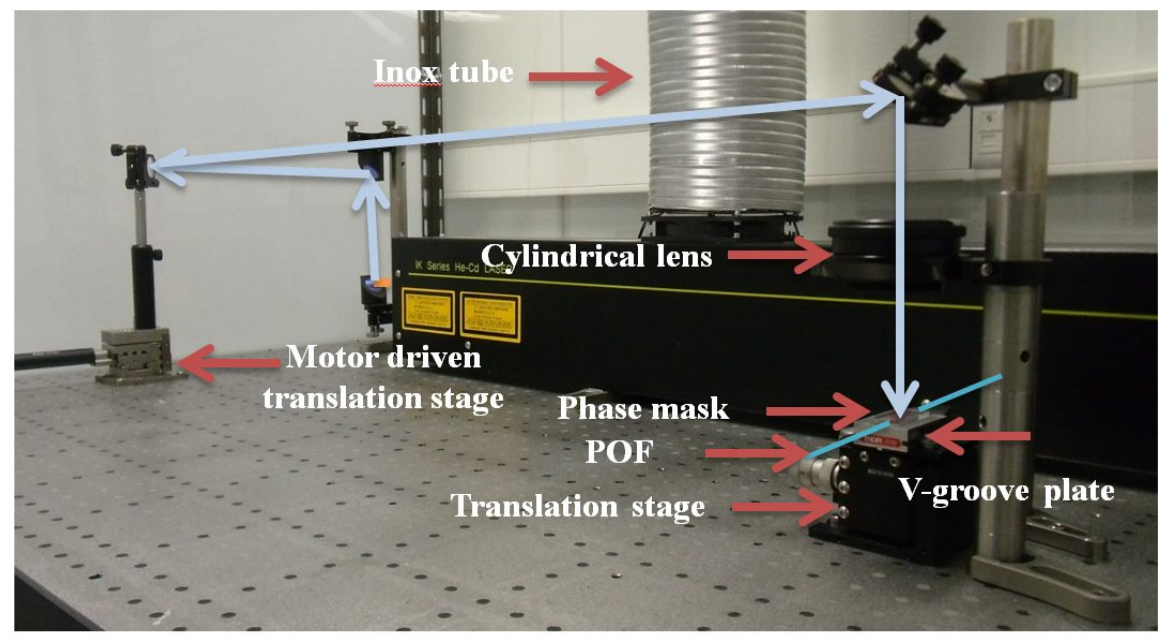

Figure 1. Experimental set-up for FBG photo-inscription in step-index POFs.

\section{PDL AND DGD MEASUREMENTS FOR UV-INDUCED FBGS}

\subsection{PDL and DGD measurements for step-index POFBGs}

The reflective FBGs in POF were measured in transmission with polarized light. We have used an optical vector analyzer (OVA CTe from Luna Technologies) that was chosen for both its high wavelength resolution (1.25 pm) and its fast scanning rate (less than $1 \mathrm{~s}$ to analyze a range of a few tens of $\mathrm{nm}$ ). Figure 2 depicts the spectra of transmission, PDL and DGD measured by the OVA. By measuring the transmitted amplitude spectrum, the maximum reflectivity has been 
computed equal to $97 \%$. The maximum value of PDL is equal to $0.9 \mathrm{~dB}$ above noise level. The latter is close to $1 \mathrm{~dB}$ and is essentially due to the splices. The photo-induced birefringence value is estimated through a numerical fit of the experimental data, using an in-house developed inverse scattering program based on the Nelder-Mead simplex algorithm ${ }^{27}$. The birefringence has been estimated to $7 \times 10^{-6}$. The maximum value of DGD is $3.3 \mathrm{ps}$ above noise level. Thus, this measurement confirms that the side inscription process induces non-negligible photo-induced birefringence in step-index POFBGs, as in the case of silica fibers ${ }^{25,26}$.

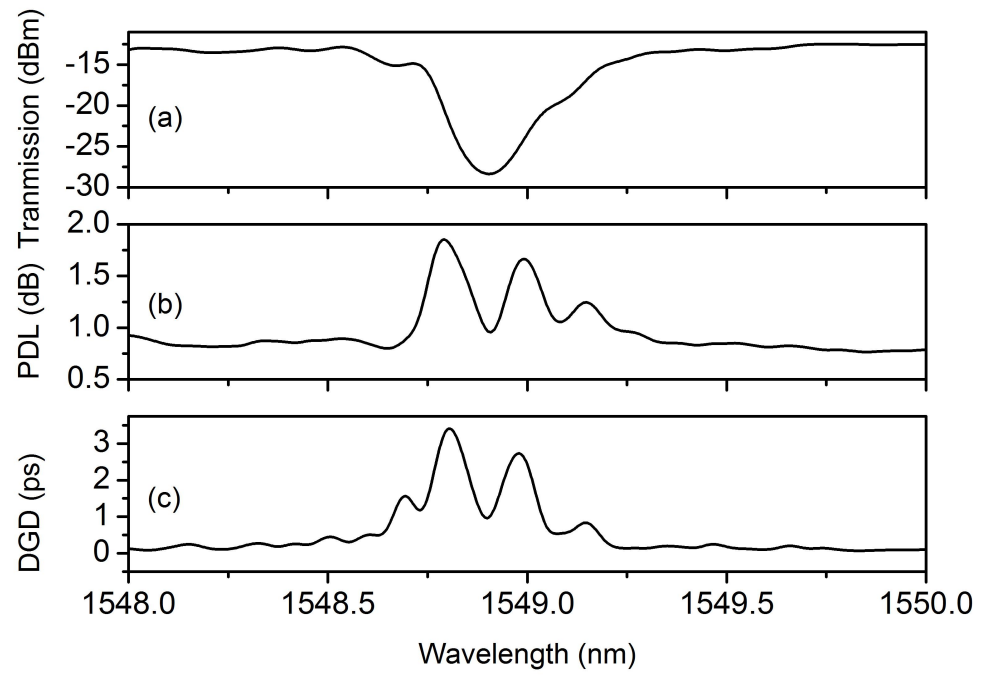

Figure 2. Transmitted spectrum (a), polarization dependent loss (b) and differential group delay (c) for a $6 \mathrm{~mm}$ long stepindex POFBG.

\subsection{PDL and DGD measurements for mPOFBGs}

Similar measurements were conducted for mPOFBGs. Figure 3 depicts the transmitted spectrum, PDL and DGD curves of a grating with a reflectivity of $86 \%$. Here, the maximum values of PDL and DGD are $4.8 \mathrm{~dB}$ and 9 ps above noise level, respectively, confirming that photo-induced birefringence is also present for this $\mathrm{mPOF}$. The irregular evolution at the left side of the rejection band is attributed to the fibre being few-moded in this wavelength range.

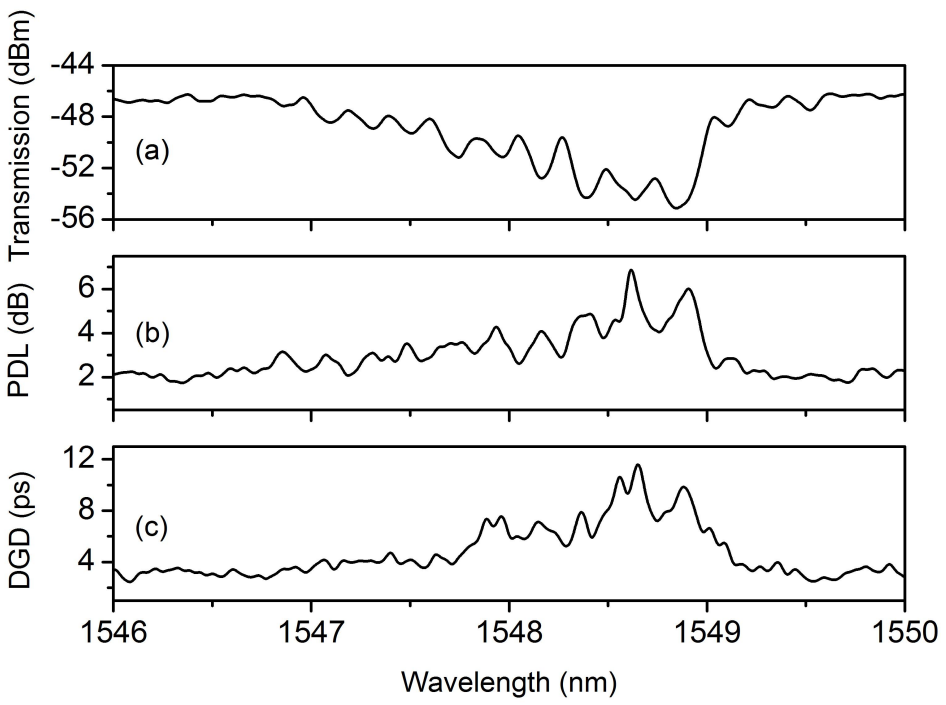

Figure 3. Transmitted spectrum (a), polarization dependent loss (b) and differential group delay (c) for a $5 \mathrm{~mm}$ long mPOFBG. 


\section{TRANSVERSE FORCE MEASUREMENT}

In this section, we make use of the PDL values for transverse force sensing purposes. In our set-up, two $20 \mathrm{~mm}$ long POFs with and without grating, respectively, were placed parallel and pressed between two metal plates, which avoided the tilt of the loading area. The loads were applied and varied on the top of the plate in the range $(0-0.75 \mathrm{~N})$ for each fiber. Figure 4 depicts the evolution of the PDL maximum value as a function of the transverse force. It can be seen that the evolution is linear in that range. A linear regression of the raw data yields a maximum PDL value sensitivity equal to $1.7 \mathrm{~dB} / \mathrm{N} \pm 0.2 \mathrm{~dB} / \mathrm{N}$.

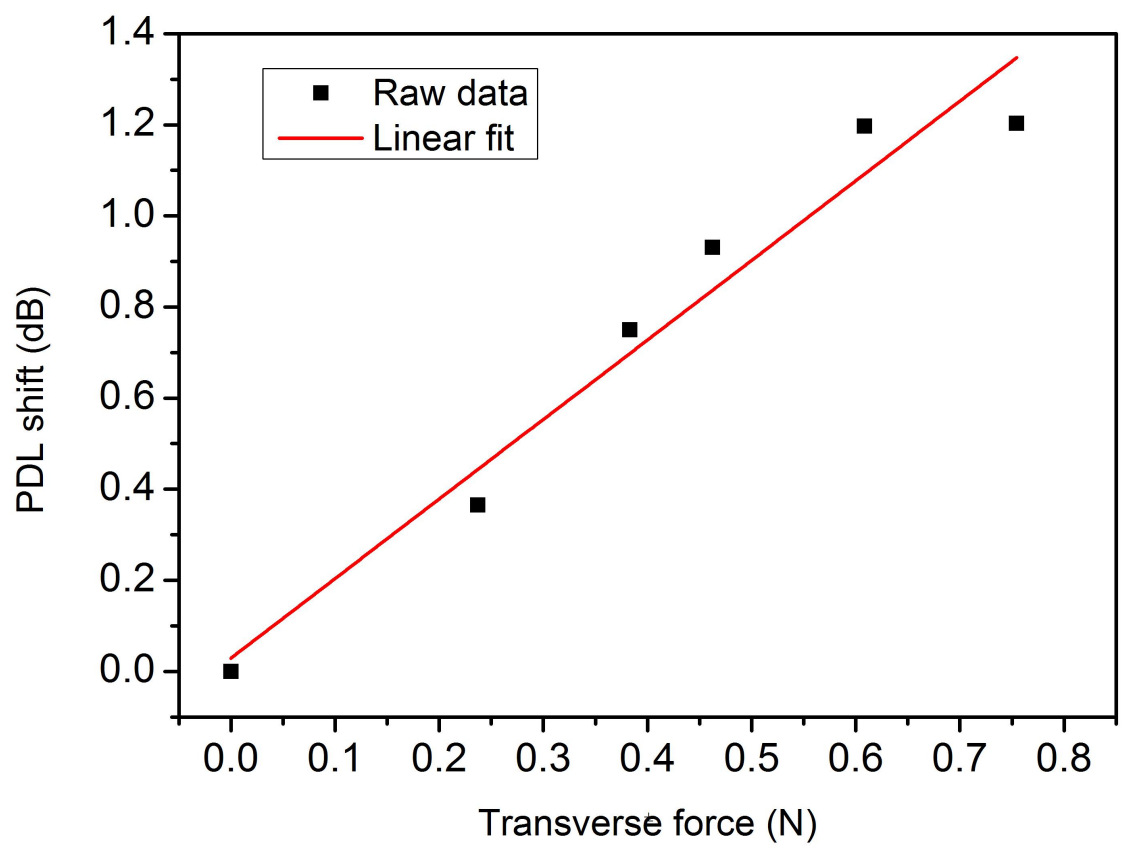

Figure 4. PDL evolution as a function of transverse force for a $6 \mathrm{~mm}$ long step-index POFBG.

\section{CONCLUSION}

(M)POFBGs were photo-written by UV laser at the wavelength of $325 \mathrm{~nm}$. Then the transmitted amplitude spectra, PDL and DGD curves were measured by an optical vector analyzer. For step-index POFBGs, the reflectivity is $97 \%$ and the maximum values of PDL and DGD are equal to $0.9 \mathrm{~dB}$ and $3.3 \mathrm{ps}$, respectively. By transverse force measurement, the PDL sensitivity is calculated to be $1.7 \mathrm{~dB} / \mathrm{N}$. As for mPOFBGs, the reflectivity is $86 \%$ and the maximum values of PDL and DGD are equal to $4.8 \mathrm{~dB}$ and $9 \mathrm{ps}$, respectively. This preliminary study about birefringence effects in POFBGs confirms the great potential that such measurements can have for transverse strain sensing.

\section{ACKNOWLEDGMENTS}

Authors gratefully thank Professor Tam from Hong Kong Polytechnic Institute for providing the optical fiber used in this work. This research has been conducted in the frame of the ERC (European Research Council) Starting Independent Researcher Grant PROSPER (grant agreement N ${ }^{\circ} 280161$ - http://www.umons.ac.be/erc-prosper) and the Actions de la Recherche Concertées research programme (PREDICTION project) supported by the Ministère de la Communauté française de Belgique_Direction générale de l'Enseignement non obligatoire et de la Recherche scientifique. C. Caucheteur is supported by the F.R.S.-FNRS. This work was partially supported by a Marie Curie Intra European Fellowship included in the 7th Framework Program of the European Union (project PIEF-GA-2011-302919). 


\section{REFERENCES}

[1] Xiong, Z., Peng, G. D., Wu, B., Chu, P. L., "Highly tunable Bragg gratings in single-mode polymer optical fibers," IEEE Photonics Technology Letters 11(3), 352-354 (1999).

[2] Stefani, A., Stecher, M., Town, G. E., Bang, O., "Direct writing of fiber Bragg grating in microstructured polymer optical fiber," IEEE Photonics Technology Letters 24(13), 1148-1150 (2012).

[3] Webb, D. J., Kalli, K., Carroll, K., Zhang, C., Komodromos, M., Argyros, A., Large, M., Emiliyanov, G., Bang, O., Kjaer, E., "Recent developments of Bragg gratings in PMMA and TOPAS polymer optical fibers," Proc. SPIE 6830, 683002 (2007).

[4] Statkiewicz-Barabach, G., Tarnowski, K., Kowal, D., Mergo, P., Urbanczyk, W., "Fabrication of multiple Bragg gratings in microstructured polymer fibers using a phase mask with several diffraction orders," Optics Express 21(7), 8521-8534 (2013).

[5] Dobb, H., Webb, D. J., Kalli, K., Argyros, A., Large, M. C. J., van Eijkelenborg, M. A., "Continuous wave ultraviolet light-induced fiber Bragg gratings in few- and single-mode microstructured polymer optical fibers," Optics Letters, 30(24), 3296-3298 (2005).

[6] Chen, X., Zhang, C., Webb, D. J., Peng, G. D., Kalli, K., "Bragg grating in a polymer optical fibre for strain, bend and temperature sensing," Measurement Science and Technology 21(9), 094005 (2010).

[7] Marques, C. A. F., Bilro, L. B., Alberto, N. J., Webb, D. J., Nogueira, R. N., "Inscription of narrow bandwidth Bragg gratings in polymer optical fibers," Journal of Optics 15(7), 075404 (2013).

[8] Yuan, W., Stefani, A., "Tunable polymer fiber Bragg grating (FBG) inscription: fabrication of dual-FBG temperature compensated polymer optical fiber strain sensors," IEEE Photonics Technology Letters 24(5), 401403 (2012).

[9] Carroll, K. E., Zhang, C., Webb, D. J., Kalli, K., Argyros, A., Large, M. C. J., “Thermal response of Bragg gratings in PMMA microstructured optical fibers," Optics Express 15(14), 8844-8850 (2007).

[10] Liu, H. Y., Peng, G. D., Chu, P. L., “Thermal tuning of polymer optical fiber Bragg gratings," IEEE Photonics Technology Letters 13(8), 824-826 (2001).

[11] Yuan, W., Stefani, A., Bache, M., Jacobsen, Rose, T., B., Herholdt-Rasmussen, N., Nielsen, F. K., Andresen, Sørensen, S., O. B., Hansen, K. S., Bang, O., "Improved thermal and strain performance of annealed polymer optical fiber Bragg gratings," Optics Communications 284(1), 176-182 (2011).

[12] Zhang, Z. F., Zhang, C., Tao, X. M., Wang, G. F., Peng, G. D., "Inscription of polymer optical fiber Bragg grating at $962 \mathrm{~nm}$ and its potential in strain sensing," IEEE Photonics Technology Letters 22(21), 1562-1564 (2010).

[13] Hu, X., Kinet, D., Chah, K., Mégret, P., Caucheteur, C., "Bragg gratings inscription at $1550 \mathrm{~nm}$ in photosensitive step-index polymer optical fiber," Proc. SPIE 8794, 87942Q (2013).

[14]Zhang, Z. F., Tao, X.M., "Synergetic effects of humidity and temperature on PMMA based fiber Bragg gratings," Journal of Lightwave Technology 30(6), 841-845 (2012).

[15] Zhang, W., Webb, D., Peng, G., "Polymer optical fiber Bragg grating acting as an intrinsic biochemical concentration sensor," Optics Letters 37(8), 1370-1372 (2012).

[16] Zhang, C., Chen, X., Webb, D. J., Peng, G. D., "Water detection in jet fuel using a polymer optical fibre Bragg grating," Proc. SPIE 7503, 750380 (2009).

[17] Yuan, W., Khan, L., Webb, D. J., Kalli, K., Rasmussen, H. K., Stefani, A., Bang, O., "Humidity insensitive TOPAS polymer fiber Bragg grating sensor," Optics Express 19(20), 19731-19739 (2011).

[18] Markos, C., Stefani, A., Nielsen, K., Rasmussen, H. K., Yuan, W., Bang, O., "High-Tg TOPAS microstructured polymer optical fiber for fiber Bragg grating strain sensing at 110 degrees," Optics Express 21(4), 4758-4765 (2013).

[19] Johnson, I. P., Yuan, Stefani, W., Nielsen, Rasmussen, A., Khan, K., H., L., Webb, D. J., Kalli, K., Bang, O., "Optical fibre Bragg grating recorded in TOPAS cyclic olefin copolymer," IEEE Electronics Letters 47(4), 271272 (2011).

[20] Sáez-Rodríguez, D., Nielsen, K., Rasmussen, H. K., Bang, O., Webb, D. J.,” Highly photosensitive polymethyl methacrylate microstructured polymer optical fiber with doped core," Optics Letters 38(19), 3769-3772 (2013).

[21] Liu, H. Y., Liu, H. B., Peng, G. D., Chu, P. L., "Observation of type I and type II gratings behavior in polymer optical fiber," Optics Communications 220(4), 337-343 (2003). 
[22] Liu, H. B., Liu, H. Y., Peng, G. D., Chu, P. L., "Novel growth behaviors of fiber Bragg gratings in polymer optical fiber under UV irradiation with low power," IEEE Photonics Technology Letters 16(1), 159-161 (2004).

[23] Belhadj, N., LaRochelle, S., Dossou, K., "Form birefringence in UV-exposed photosensitive fibers computed using a higher order finite element method," Optics Express 12(8), 1720-1726 (2004).

[24] Erdogan, T., Mizrahi, V., "Characterization of UV-induced birefringence in photosensitive Ge-doped silica optical fibers," Journal of Optical Society America B 11(10), 2100-2105 (1994).

[25] Caucheteur, C., Bette, S., Garcia-Olcina, R., Wuilpart, M., Sales, S., Capmany, J., Mégret, P., "Transverse strain measurements using the birefringence effect in fiber Bragg gratings," IEEE Photonics Technology Letters 19(13), 966-968 (2007).

[26] Caucheteur, C., Bette, S., Garcia-Olcina, R., Wuilpart, M., Sales, S., Capmany, J., Mégret, P., "Influence of the grating parameters on the polarization properties of fiber Bragg gratings," Journal of Lightwave Technology 27(8), 1000-1010 (2009).

[27]Lhommé, F., Caucheteur, C., Chah, K., Blondel, M., Mégret, P., "Synthesis of fiber Bragg grating parameters from experimental reflectivity: a simplex approach and its application to the determination of temperaturedependent properties," Applied Optics 44(4), 493-497 (2005). 